\title{
Tumefactive Multiple Sclerosis Clinically Mimicking Acute Stroke and Lesional Migration Along the Biopsy Tract: A Case Report
}

\author{
Oranan Tritanon, MD. ${ }^{1}$, Arunee Singhsnaeh, MD. ${ }^{2 *}$, Jiraporn Laothamatus, MD. ${ }^{1,3}$, \\ Atthaporn Boongird, MD. ${ }^{4}$ Disya Ratanakorn, MD. ${ }^{5}$, Prawat Nitiyanant, MD. ${ }^{2}$ \\ Division of Diagnostic Neuroradiology, Department of Diagnostic and Therapeutic Radiology, \\ ${ }^{2}$ Department of Pathology, ${ }^{3}$ Advanced Diagnostic Imaging Center, \\ ${ }^{4}$ Division of Neurosurgery, Department of Surgery, ${ }^{5}$ Division of Neurology, Department of Internal Medicine, \\ Faculty of Medicine Ramathibodi Hospital, Mahidol University, Bangkok, Thailand
}

\begin{abstract}
Tumefactive multiple sclerosis is a form of demyelinating disease which patient can present with acute stroke. We reported a case of a 49-year-old woman with well controlled hypertension, who presented with right hemiplegia 15 hours prior to admission. The initial diagnosis of acute stroke was made. Emergency computed tomography showed hypodense lesion at the left lentiform nucleus and posterior limb of the left internal capsule. The magnetic resonance imaging (MRI) study showed hyperintense FLAIR lesion in the left lentiform nucleus, left internal capsule, left thalamus, and periventricular area of the left frontoparietal region, some areas of restricted diffusion and inhomogeneous enhancement. The MR spectroscopy (MRS) of the lesion showed increased choline peak, decreased creatine and NAA peaks, and maximal choline to creatine ratio 2.25 . Her symptoms deteriorated with progressive headache and motor aphasia. The follow up MRI showed extension of the inhomogeneous enhancing lesion along the biopsy tract at the left frontal lobe with the enhancing and MR spectra pattern similar to the lesion.

The craniotomy with left frontal lesion excision included the mass and the biopsy tract was done. The lesion showed acute and chronic inflammatory cell infiltration with macrophages, necrotic tissue and reactive gliosis. The further pathological worked up demonstrated foci of demyelination with relative axonal preservation, numerous CD68+ macrophages with intracyto-plasmic Luxol fast blue(+) myelin debris. Perivascular and parenchymal CD3+ T-cells were identified, especially in demyelinating foci. These findings supported the diagnosis of tumefactive multiple sclerosis.

Her conditions were improved after treating with pulse methylprednisolone and intravenous immunoglobulin (IVIG). Follow up MRI study 4 months after treatment revealed almost resolution of the preexisting inhomogeneous enhancing lesion.
\end{abstract}

* Corresponding author: Arunee Singhsnaeh, email: arueed@gmail.com 


\section{Introduction}

Tumefactive multiple sclerosis is often described as atypical feature of multiple sclerosis that is larger than $2 \mathrm{~cm}$, mass effect, oedema and/or ring enhancement, which clinically and radiographically mimicking stroke, intracranial tumor and infection $^{(1,2)}$. We reported a case of biopsy-proven tumefactive MS patient that clinically mimicking acute stroke and radiographically mimicking intracranial lymphoma that challenged neurologist and radiologist.

\section{Case reports}

A 49-year old woman with well controlled hypertension presented with acute right upper and lower extremities weakness 15 hours prior to hospitalization. Physical examination showed right hemiparesis, right facial palsy upper motor neuron type, positive right Babinski reflex, positive jaw jerk reflex, and motor power grade I on the right extremities.

CT scan of the brain at emergency room showed hypodense lesion at the left lentiform nucleus and posterior limb of the left internal capsule (Fig 1A).

First MRI of the brain in two days later disclosed the lesion seen as hyperintense T2W and FLAIR in the left lentiform nucleus, genu \& posterior limb of the left internal capsule, left thalamus, left corona radiata, left cerebral peduncle and periven-

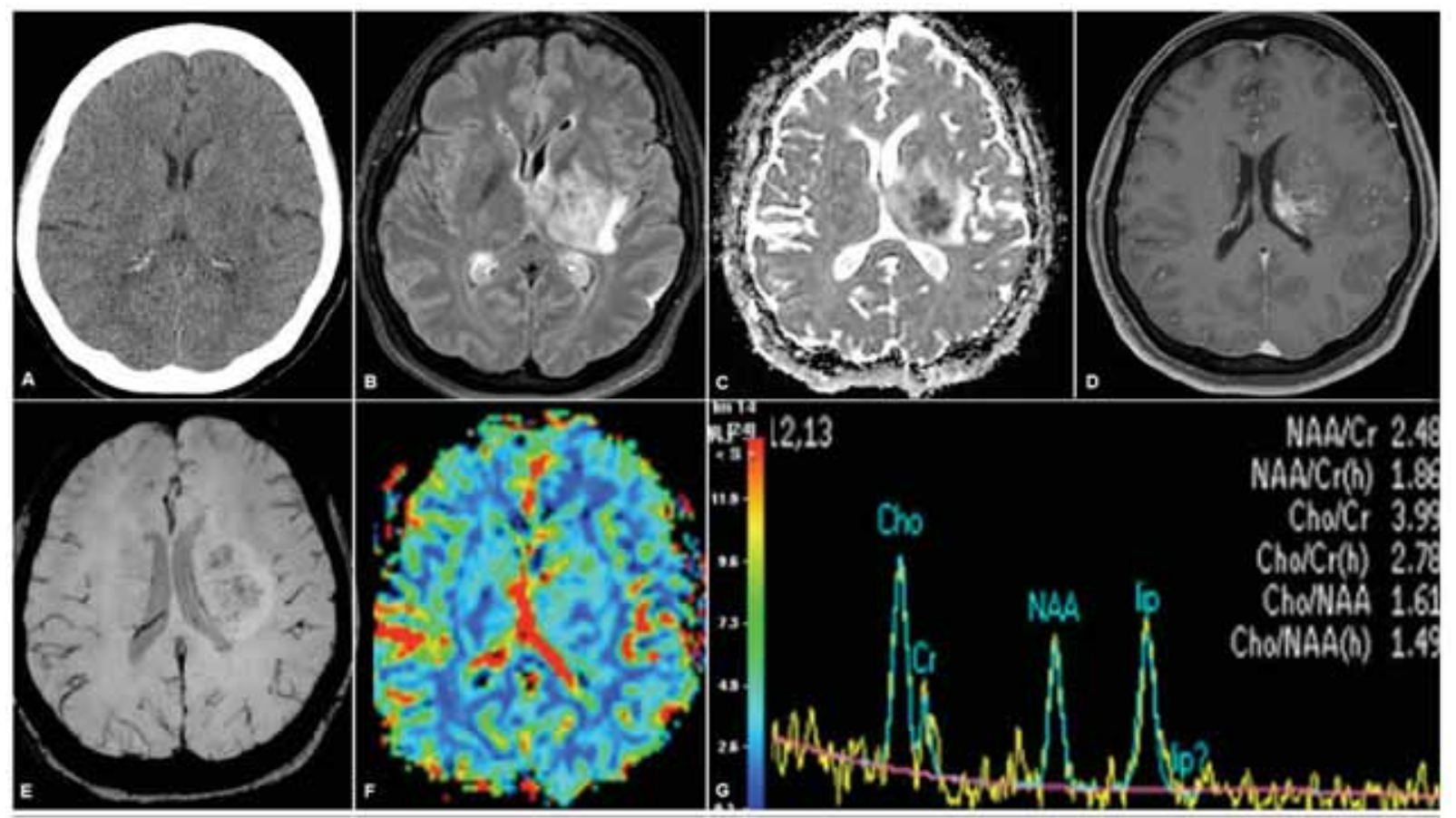

Fig 1. (A) Non contrast CT scan at initial presentation showed hypodense lesion at the left lentiform nucleus and posterior limb of the left internal capsule. (B) FLAIR MRI showed hyperintense at the left lentiform nucleus, genu \& posterior limb of the left internal capsule, left thalamus and left external capsule. (C) ADC showed the area of restricted diffusion within the lesion. (D) Gd-THRIVE showed inhomogeneous enhancing and nonenhancing areas. (E) SWI showed prominent parallel venules within the lesion. (F) Perfusion images revealed increased CBF of the lesion. (G) MRS revealed increased choline peak, decreased creatine and NAA peaks. 
tricular area of the left frontoparietal region with prominent perivenular space within the lesion (Fig 1B). The area of restricted diffusion within the lesion was detected (Fig 1C). The inhomogeneous enhancing and non-enhancing areas on axial GdTHRIVE were noted, which demonstrated intense patchy enhancement at the periventricular area (Fig 1D). In SWI, there were prominent parallel venules within the lesion radial to the body of left lateral ventricle (Fig 1E). Also, there were the increased cerebral blood flow (CBF) and cerebral blood volume (CBV) of the lesion in MR perfusion (Fig 1F). There was increased choline peak, decreased creatine and NAA peaks, maximal choline to creatine ratio 2.25, maximal choline to NAA ratio 1.61 on MR spectra using multivoxel, 2000/ 144 TR/ TE) (Fig 1).

First biopsy obtained from the left thalamus revealed perivascular acute and chronic inflammatory cell infiltration in the background of reactive gliosis. Inflammation/infectious process were suspected (Fig. 2). Special stains for acid fast bacilli and fungi showed negative findings.

Two week later, the patient had progressive headache and motor aphasia. The $2^{\text {nd }}$ MRI showed an increased enhancing area within the preexisting lesion (Fig 3). Also seen was an extension of the enhancing lesion along the biopsy tract at the left frontal lobe with the enhancing and MR spectra pattern similar to the lesion (Fig 4, 5).

The $2^{\text {nd }}$ biopsy obtained from the left frontal lobe and tissue from biopsy tract also showed similar pathologic findings that were acute and chronic inflammatory cell infiltration along perivascular area in background of gliosis and numerous foamy histiocytes.

Because of suspicious of neoplastic process and deterioration of clinical conditions, the craniotomy with left frontal lesion removal of the mass and biopsy tract was done. The lesion showed more acute and chronic inflammatory cell infiltration with marked increased macrophages, necrotic tissue and reactive gliosis (Fig. 6). The further pathological worked up demonstrated foci of demyelination with relative axonal preservation, numerous CD68+ macrophages with intracyto-plasmic Luxol fast blue(+) myelin debris. (Fig 7) Perivascular and parenchymal CD3+ T-cells were identified especially in demyelinating foci. These findings supported the diagnosis

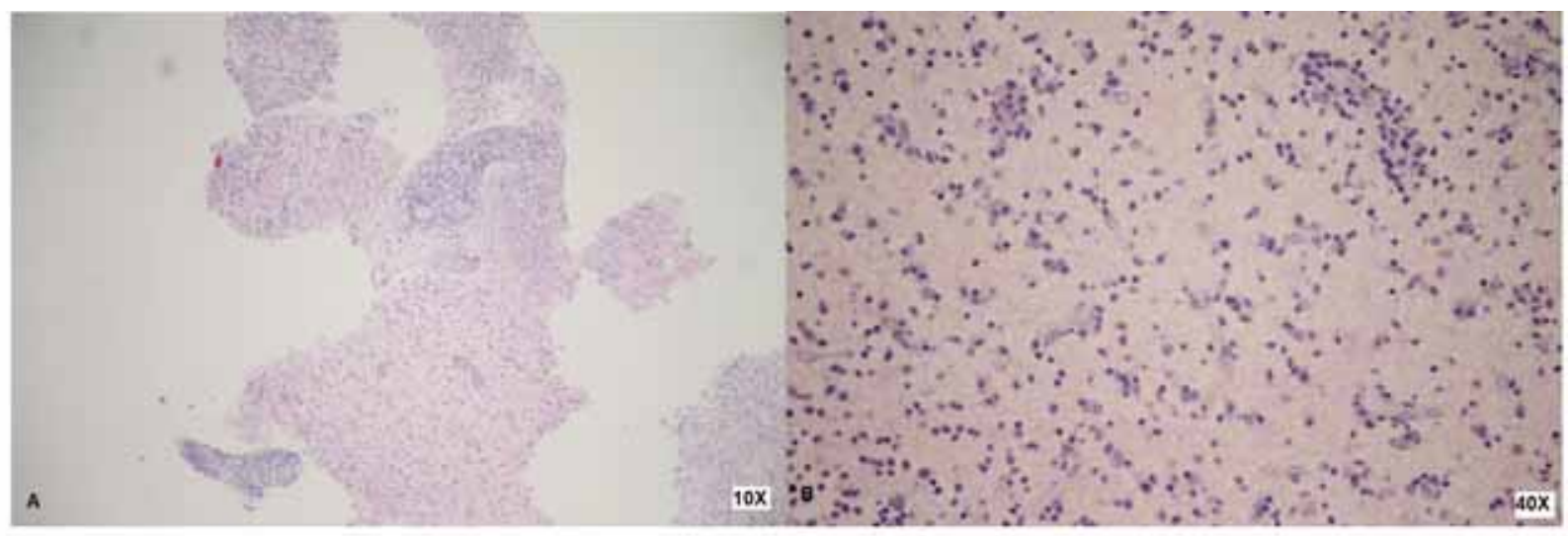

Fig 2. $(A, B)$ Perivascular and intraparenchymal acute and chronic inflammatory cell infiltration 


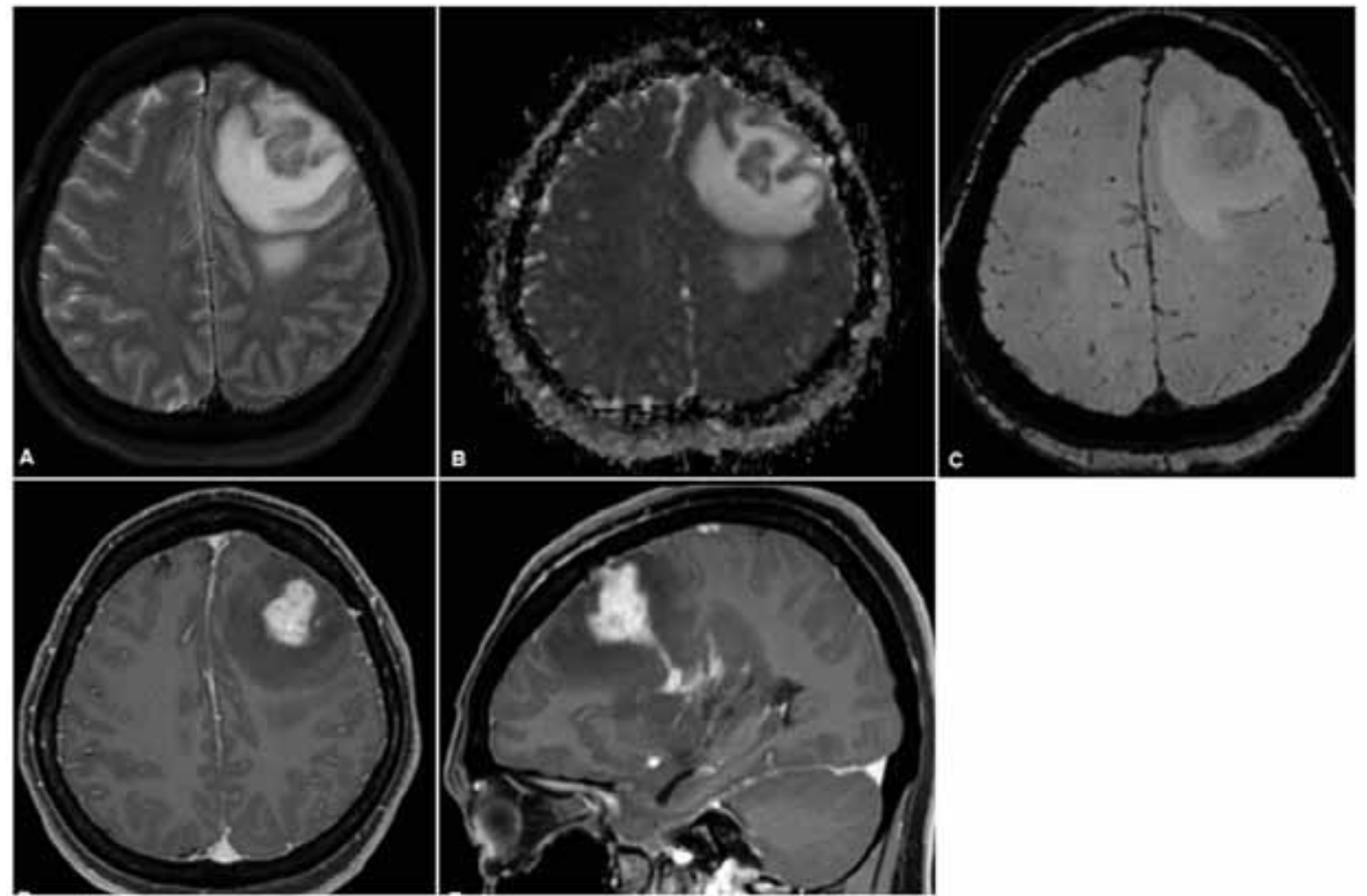

Fig 3. Two weeks later, follow up MRI of the brain

(A) FLAIR MRI revealed increased hyperintense area of the preexisting lesion. (B) Gd-THRIVE showed increased enhancing area within the preexisting lesion
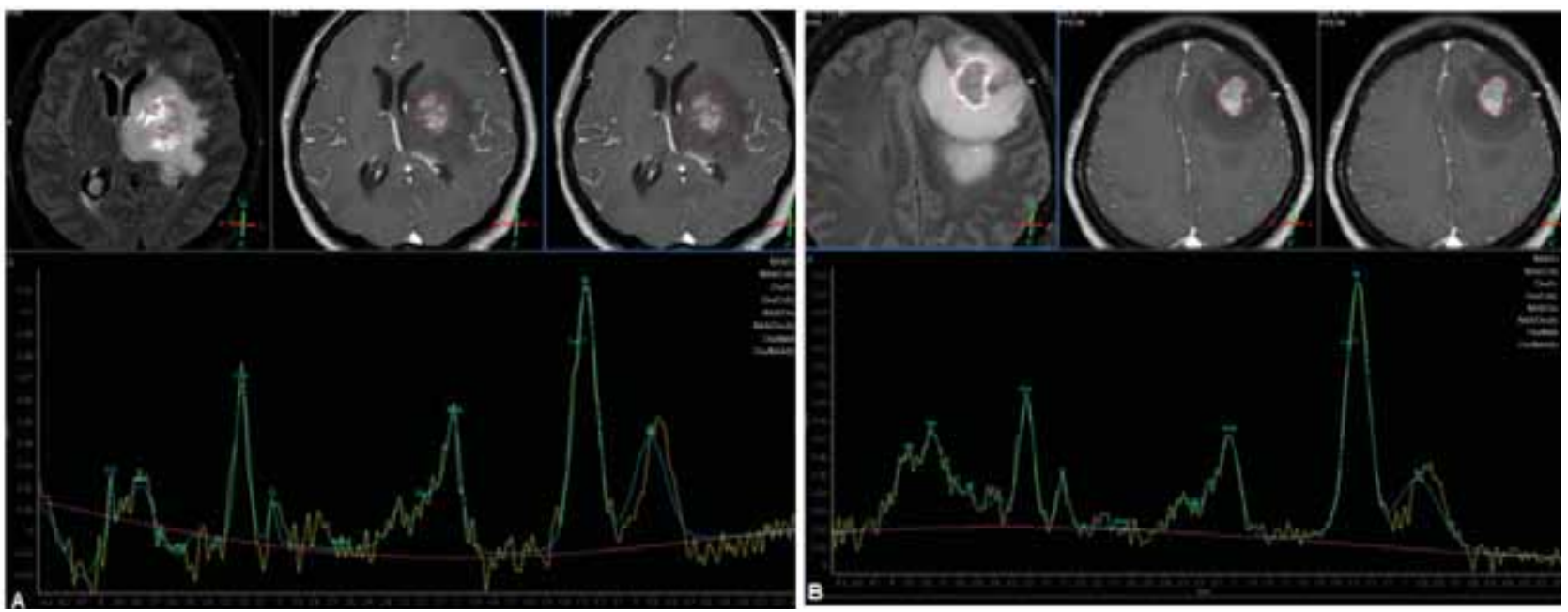

Fig 5. (A, B) MRS showed similar pattern of the lesion along the biopsy tract at the left frontal lobe and the left lentiform nucleus 


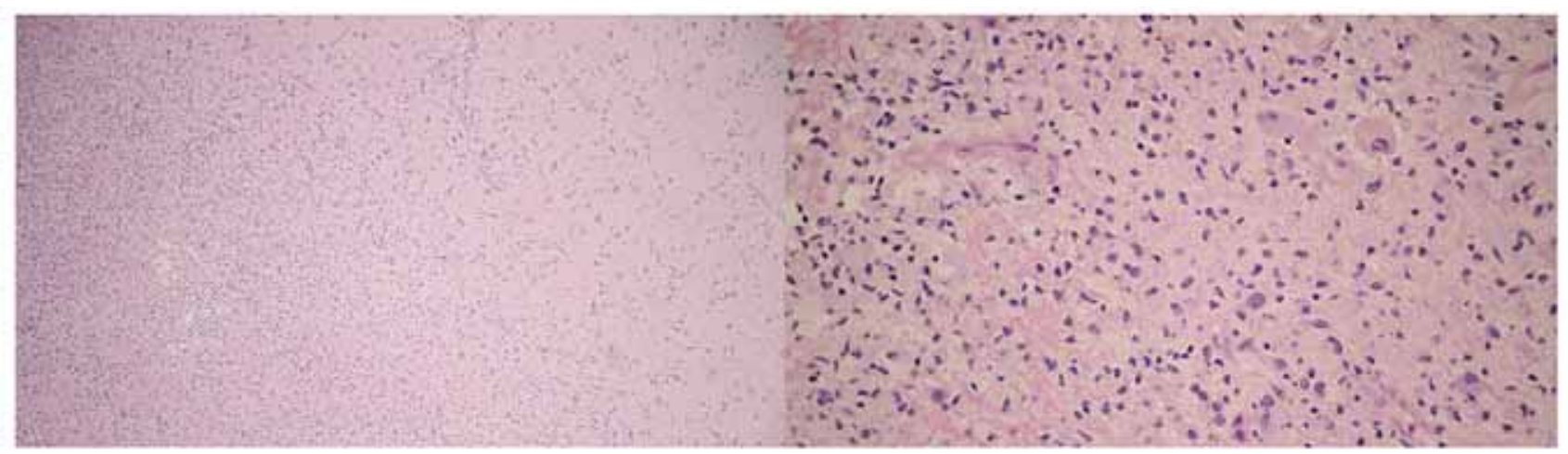

Fig 6. More acute and chronic inflammation with numerous macrophage infiltrations

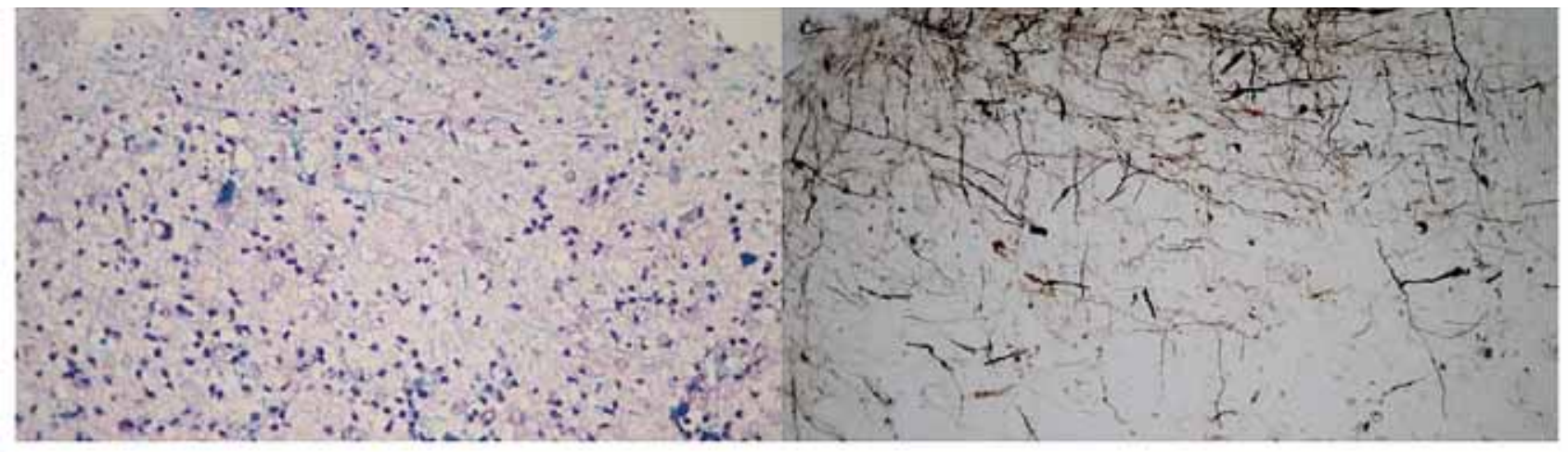

Fig 7. Luxol fast blue PAS and Bielschowsky staining show demyelination with axonal preservation

of tumefactive multiple sclerosis.

Her clinical improved after treatment with pulse methylprednisolone and intravenous immunoglobulin (IVIG). Follow up MRI studies 4 months after treatment revealed almost resolution of the preexisting inhomogeneous enhancing lesion that known as tumefactive multiple sclerosis.

\section{Discussion}

Multiple sclerosis is an inflammatory demyelinating condition attacking the myelin that causes absence of proteolipid protein in area of demyelination $^{(3)}$. Tumefactive MS is atypical feature of multiple sclerosis that sometimes mimic intracranial tumor such as lymphoma or glioblastoma multiforme $(\mathrm{GBM})^{(3)}$. In our case, there were some characters on MR perfusion and MR spectroscopy which showed markedly increased CBF and CBV, increased choline peak, choline to NAA and choline to creatine ratio. These findings made the difficulty to exclude high grade brain tumor. However prominent venules in SWI and dilated perivenular space on $\mathrm{T} 2 \mathrm{~W}$ in our case were the clues for diagnosis of tumefactive MS, that represented inflammatory activity and perivenular lymphocytic cuffing ${ }^{(4,5)}$.

Interestingly, there was an extension of the demyelinating lesion along the biopsy tract which sometimes confused with the tumor seeding. The 
pathological-proven this migratory lesion along the biopsy tract confirmed the same demyelination as the basal ganglion \& thalamic lesion. These findings may be explained by the activation of immune \& inflammatory process along the biopsy tract.

\section{Conclusion}

Tumefactive multiple sclerosis is "tumor-like" lesion and radiologically mimicking tumors, which can infiltrates along the biopsy tract confusing with tumor seeding. Prominent venules in SWI and dilated perivenular space on T2W are helpful signs for diagnosis. Biopsy is required, if there are atypical clinical presentations and MR imaging. Luxol fast blue PAS and Bielschowsky stains should be considered if tumefactive multiple sclerosis is suspected.

\section{References}

1. Lucchinetti CF, Gavrilova RH, Metz I, Parisi JE, Scheithauer BW, Weigand S. Clinical and radiographic spectrum of pathologically confirmed tumefactive multiple sclerosis. Brain 2008;131:1759-75.

2. Yacoub HA, Al-Qudahl ZA, Lee HJ, Baisre A, Souayah N. Tumefactive multiple sclerosis presenting as acute ischemic stroke. Journal of Vascular and Interventional Neurology 2011;4(2):21-3.

3. Trapp BD, Peterson J, Ransohoff RM, Rudick R, Mörk S, $B o ̈ L$. Axonal transection in the lesions of multiple sclerosis. The New England Journal of Medicine 1998; 338(5): 278-85.

4. Ge Y, Law M, Herbert J, Grossman RI. Prominent perivenular spaces in multiple sclerosis as a sign of perivascular inflammation in primary demyelination. AJNR 2005; 26:2316-9.

5. Given CA, Stevens BS, Lee C. The MRI appearance of tumefactive demyelinating lesions. AJR 2004;182:195-9. 